OPEN ACCESS

Edited by: Yingying Qin,

Shandong University, China

Reviewed by:

Lara Tamburrino,

University of Florence, Italy

Necati Findikli,

Bahçeci Fulya IVF Center, Turkey

${ }^{*}$ Correspondence:

Qing Xue

drxueqing@163.com

Specialty section: This article was submitted to

Reproduction,

a section of the journal

Frontiers in Endocrinology

Received: 30 May 2021 Accepted: 02 August 2021

Published: 23 August 2021

Citation:

Li F, Lu R, Zeng C, Li X and Xue Q (2021) Development and Validation of a Clinical Pregnancy Failure Prediction Model for Poor Ovarian Responders During IVF/ICSI.

Front. Endocrinol. 12:717288. doi: 10.3389/fendo.2021.717288

\section{Development and Validation of a Clinical Pregnancy Failure Prediction Model for Poor Ovarian Responders During IVF/ICSI}

\author{
Fangyuan Li, Ruihui Lu, Cheng Zeng, Xin Li and Qing Xue* \\ Department of Gynecology and Obstetrics, Peking University First Hospital, Beijing, China
}

Backgrounds: Despite the great advances in assisted reproductive technology (ART), poor ovarian response (POR) is still one of the most challenging tasks in reproductive medicine. This predictive model we developed aims to predict the individual probability of clinical pregnancy failure for poor ovarian responders (PORs) under in vitro fertilization/ intracytoplasmic sperm injection (IVF/ICSI).

Methods: The nomogram was developed in 281 patients with POR according to the Bologna criteria from January 2016 to December 2019, with 179 in the training group and 102 in the validation group. Univariate and multivariate logistic regression analyses were used to identify characteristics that were associated with clinical pregnancy failure. The nomogram was constructed based on regression coefficients. Performance was evaluated using both calibration and discrimination.

Results: Age $>35$ years, body mass index $(\mathrm{BMI})>24 \mathrm{~kg} / \mathrm{m}^{2}$, basic follicle-stimulating hormone $(\mathrm{FSH})>10 \mathrm{mlU} / \mathrm{ml}$, basic $\mathrm{E} 2>60 \mathrm{pg} / \mathrm{ml}$, type $\mathrm{B}$ or $\mathrm{C}$ of endometrium on human chorionic gonadotropin ( $\mathrm{hCG}$ ) day, and the number of high-quality embryos $<2$ were associated with pregnancy failure of POR patients. The area under the receiver operating characteristic curve (AUC) of the training set is 0.786 (95\% confidence interval (Cl): 0.710 0.861), and AUC in the validation set is $0.748(95 \% \mathrm{Cl}$ : 0.668-0.827), showing a satisfactory goodness of fit and discrimination ability in this nomogram.

Conclusion: Our nomogram can predict the probability of clinical pregnancy failure in PORs before embryo transfer in IVF/ICSI procedure, to help practitioners make appropriate clinical decisions and to help infertile couples manage their expectations.

Keywords: predictive model, clinical pregnancy failure, poor ovarian response, IVF/ICSI, nomogram

\section{INTRODUCTION}

With the development of in vitro fertilization/intracytoplasmic sperm injection (IVF/ICSI) techniques over the past decades, the purpose of personalized treatment of IVF/ICSI is to help every couple maximize the chances of pregnancy and eliminate the avoidable risks resulting from ovarian stimulation (1). Despite the advances in assisted reproductive technology (ART), poor 
ovarian response (POR) poses a great challenge in that the number of oocytes collected is usually below expectation with the appropriate ovarian stimulation (2-4), leading to fewer transferable embryos, greater odds for cycle cancellation, and lower pregnancy rates (as low as 2\%-4\%) (5-7). The incidence of poor ovarian responders (PORs) among infertile women has been reported to vary between $5.6 \%$ and $35.1 \%(8-11)$, and POR affects approximately $11.9 \%$ women in China undergoing IVF treatment (12). Therefore, POR is considered as one of the success-limiting factors for IVF/ICSI outcomes (13).

As ART treatment is expensive, invasive, and not a guarantee of success, infertile couples need to be informed about their chances of pregnancy to manage their expectations. Although some studies have used multivariate regression models to identify predictive factors associated with IVF/ICSI outcomes (14-16), none of them targeted evaluating the chances of clinical pregnancy in PORs.

Therefore, we aimed to evaluate the risk of poor pregnancy outcomes and develop a nomogram to predict the probability of clinical pregnancy failure in patients with POR before embryo transfer in IVF/ICSI procedure, in order to make appropriate clinical decisions and help couples manage their expectations.

\section{MATERIALS AND METHODS}

\section{Patients' Selection}

Patients who underwent IVF/ICSI-embryo transfer (IVF/ICSI-ET) cycles between January 2016 and December 2019 were retrospectively reviewed in the Department of Obstetrics and Gynecology, Peking University First Hospital. All women were routinely requested for their data to be used for research purposes, and those who refused consent were excluded from this study. The local ethics committee granted permission to this study.

Patients were eligible if they fulfilled the definition of POR according to the Bologna criteria (17) - at least two of the following three features must be present: 1 ) advanced maternal age ( $\geq 40$ years) or any other risk factors for POR (evidence of ovarian cysts, previous ovarian surgery, previous chemotherapy, and shortening of the menstrual cycle); 2) a previous POR cycle ( $\leq 3$ oocytes retrieved or a previous cycle canceled because of $\leq 3$ developing follicles with a conventional stimulation protocol using at least $150 \mathrm{IU}$ follicle-stimulating hormone (FSH) per day); 3) decreased ovarian reserve [i.e., antral follicle count (AFC) $<7$ follicles or anti-Müllerian hormone $(\mathrm{AMH})<1.1$ $\mathrm{ng} / \mathrm{ml}]$.

The exclusion criteria were as follows: hydrosalpinx, uterine fibroids, adenomyosis, uterine malformations, intrauterine adhesion, recurrent spontaneous abortion, antiphospholipid syndrome, chromosome karyotype abnormality, drug allergies, mental disorders and disturbance of consciousness, and a total number of previous IVF cycles $>3$.

Finally, a total of 281 patients were included in this study. They were included only for one cycle with transfer of two fresh embryos (the first cycle after fulfilling the criteria).

\section{Controlled Ovarian Hyperstimulation and Embryo Transfer}

A long gonadotrophin $(\mathrm{Gn})$-releasing hormone $(\mathrm{GnRH})$ agonist (GnRH-a) protocol, an antagonist protocol, or a mild ovarian stimulation protocol was used for ovarian stimulation in this study.

The GnRH-a protocol consisted of daily injections of shortacting and long-acting GnRH-a at different doses during the early follicular or mid-luteal phases. For the daily short-acting GnRH-a injections, patients received an injection of $0.1 \mathrm{mg} /$ day of Decapeptyl (Ferring AG, Dübendorf, Switzerland) from the mid-luteal phase of the previous cycle and continued for approximately 15 to 18 days. After ovarian suppression, the dose of Decapeptyl was reduced to $0.05 \mathrm{mg} /$ day and continued until the day of hCG (Zhuhai Lizhu Pharmaceutical Co., Ltd., Zhuhai, China) administration. For the administration of long-acting $\mathrm{GnRH}$-a protocols, triptorelin (Ipsen Pharma Biotech, Signes, France) was injected during the early follicular period, and $\mathrm{Gn}$ was injected after 21 to 35 days.

The GnRH antagonist protocol consisted of daily Gn stimulation from days 2 to 3 of menstruation, followed by daily injections of $0.25 \mathrm{mg}$ of Cetrotide (Baxter Oncology $\mathrm{GmbH}$, Frankfurt, Germany) once the leading follicle reached $14 \mathrm{~mm}$ and until the day of hCG injection.

Regarding the mild ovarian stimulation, $\mathrm{GnRH}$ antagonist was added when the dominant follicle reached $14 \mathrm{~mm}$ and until the day of hCG injection.

The choice of protocol for ovarian stimulation was based on the patient's characteristics. When more than three leading follicles measured $18 \mathrm{~mm}$ or more, hCG was administered. Thirty-six hours later, oocyte retrieval was performed under ultrasonic guidance followed by IVF/ICSI. Embryos were transferred on day 2 or 3 . The luteal phase was supported by daily vaginal or intramuscular progesterone until 2 weeks after ET.

\section{Data Collection}

Patient clinical parameters (age, stimulation protocols, body mass index (BMI), type of infertility, hormones concentration on day 3 and on hCG day, Gn dose, type and thickness of endometrium, and clinical outcomes) were collected from our database according to the literature review and clinical experiences. Metaphase II (MII) oocytes were determined 16-18 h following retrieval for conventional IVF/ICSI cycles.

Endometrial features including endometrial thickness and pattern were assessed on the day of hCG administration under Bultrasonography. Endometrial thickness was measured in a median longitudinal plane of the uterus as the maximum distance between the endometrial-myometrial interface of the anterior to the posterior wall of the uterus. The endometrial pattern was classified as pattern A (a triple-line pattern consisting of a central hyperechoic line surrounded by two hypoechoic layers), pattern $\mathrm{B}$ (an intermediate pattern with the same reflectivity as the surrounding myometrium and a poorly defined central echogenic line), and pattern C (homogenous, hyperechogenic endometrium).

Cleavage embryos were classified as high-quality embryos (grade I and II embryos) if they had three to five cells on day 2 or seven to nine cells on day 3 and as same-sized blastomeres if with less than $20 \%$ blastomeric fragments. Embryos graded III or IV including 
those that had only two cells on day 2 , less than seven cells on day 3 , and no less than $20 \%$ fragmentation were called poor quality.

Clinical pregnancy was established by gestational sacs and embryo buds under B-ultrasonography 4 weeks after embryo transplantation.

\section{Statistical Analysis}

Patients were divided into a training set and a validation set by the sampling techniques of random numbers. Baseline characteristics of patients were expressed as descriptive statistics. Continuous variables are shown as mean \pm standard deviation (SD) (normally distributed) and median (interquartile range) (non-normally distributed). Student's t-tests (normally distributed) or the MannWhitney U-test (non-normally distributed) were used to compare variables between groups. Categorical variables are presented as percent, and the chi-squared test was used for statistical comparison of percentages. These data were analyzed with SPSS 22.0.

A univariate logistic regression analysis was used to identify predictors associated with adverse maternal outcomes. The cutoff points of candidate variables were chosen to develop the model based on clinical availability. Age $>35$ years is used to diagnose advanced maternal age. BMI greater than $24 \mathrm{~kg} / \mathrm{m}^{2}$ can be diagnosed as overweight according to the Chinese standards (18). Duration of infertility $>3$ years is considered to be associated with low pregnancy rates (19). We chose some indicators of the diminished ovarian reserve (DOR) to explore whether they could predict the clinical pregnancy in a model such as basal FSH over 10 $\mathrm{mIU} / \mathrm{ml}$, basal FSH/basal luteinizing hormone $(\mathrm{LH})>3$, basal E2 $>60 \mathrm{pg} / \mathrm{ml}$, AMH level $<0.7 \mathrm{ng} / \mathrm{ml}$, and the number of AFC $\leq 5$ (17, 20-22). Endometrial thickness $>7 \mathrm{~mm}$ and triple-line pattern are endometrial receptivity markers as prognostic factors for conceiving (23). A meta-analysis of over 60,000 fresh IVF cycles showed a decreased probability of pregnancy achievement in women with progesterone elevation (PE) on the day of hCG administration (when PE was defined using a threshold $>0.8 \mathrm{ng} / \mathrm{ml}$ ) compared with those without PE (24). The other cutoff points for the number of retrieved oocytes, MII oocytes, and high-quality embryos were based on receiver operating characteristic (ROC) analysis. We used Youden's index (25) to calculate the optimal cutoff points of the three parameters related to the occurrence of pregnancy failure. A multivariate logistic regression analysis was performed to test the independent significance of different factors. The variables with $\mathrm{p}$ values $<0.1$ in univariate analysis were included in multivariate analysis. The variables were selected by stepwise regression and then fit a more parsimonious model. Variables entered into the model were age, BMI, basal E2, basal FSH, type of endometrium on human chorionic gonadotropin (hCG) day, and the number of highquality embryos.

The area under the ROC curve (AUC) was used to evaluate the predictive accuracy. Calibration curves were assessed graphically by plotting the observed rates against the predicted probabilities to evaluate the agreement. The Brier score was used to evaluate probability calibration. Nomograms are a pictorial representation of a complex mathematical formula that uses two or more known variables to calculate an outcome. The resulting model was simplified into a nomogram to predict the possibility of clinical pregnancy failure for PORs.
The performance of the nomogram was quantified concerning discriminative power and calibration in the validation cohort for external validation. An internal validation step was performed to counteract the possible overfitting of our model to the data. The bootstrap (with 200 bootstrapped samples) was used to validate and correct the over-optimism of the models. We also did a decisioncurve analysis to assess the clinical applicability of the model.

All analyses were performed using the $\mathrm{R}$ software, version 3.6.1.

\section{RESULTS}

\section{Description of the Study Population}

After the inclusion and exclusion criteria of the current study were applied, a total of 281 POR patients who underwent the IVF/ICSI procedures from January 2016 to December 2019 were identified as eligible and were analyzed in this study. Then, the patients were divided into the training set $(n=179)$ to build the model, and the validation set $(n=102)$ to test the performance. The basic characteristics are summarized in Table 1. Except for the $\mathrm{Gn}$ days ( $\mathrm{p}=0.040$ ), no significant difference is observed in the baseline characteristics between the two groups. Sixty-one patients $(34.08 \%)$ achieved clinical pregnancy in the training set.

\section{Logistic Regression Analysis}

The univariate logistic regression analysis of pregnancy failure in the developing group is listed in Table 2 . The optimal cutoff points of the values were chosen according to the clinical consensus or ROC curve of our data. According to the univariate logistic regression analysis, values with $\mathrm{p}<0.1$ were included in the multivariate logistic regression analysis for pregnancy failure.

\section{Development of the Models From the Training Cohort}

The variables were selected by stepwise regression and then fit a more parsimonious model. Finally, as shown in Table 3, the six independent risk factors for clinical pregnancy included in the prediction model are as follows [OR (95\% CI), p-value]: age $>35$ years [2.59 (1.24-5.47), $\mathrm{p}=0.012], \mathrm{BMI}>24 \mathrm{~kg} / \mathrm{m}^{2}$ [3.22 (1.45-7.58), $\mathrm{p}=0.005]$, basic FSH $>10 \mathrm{mIU} / \mathrm{ml}$ [2.87 (1.28-6.75), $\mathrm{p}=0.012]$, basic E2 $>60 \mathrm{pg} / \mathrm{ml}[2.47(1.08-5.93), \mathrm{p}=0.036]$, type $\mathrm{B}$ or $\mathrm{C}$ of endometrium on hCG day [2.47 (1.18-5.24), $\mathrm{p}=0.017]$, and the number of high-quality embryos $<2$ [2.24 (1.02-4.96), $\mathrm{p}=0.045]$.

The nomogram of prediction is shown in Figure 1. The optimal threshold point was calculated using the ROC curve. When the total points are greater than 236.472, women with POR show a high risk for a failed pregnancy.

\section{Model Validation}

The AUC of the model in the training set (Figure 2A) is 0.786 (95\% CI: $0.710-0.861$ ), which indicated a good performance. The sensitivity is $73.1 \%$, and the specificity is $76.4 \%$. The AUC of the model in the validation set (Figure 2C) is 0.748 (95\% CI: 0.6680.827 ). The sensitivity is $69.2 \%$, and the specificity is $73.2 \%$. The slope of calibration curves in the training set (Figure 2B) and the validation set (Figure 2D) is 1.000 and 1.000, respectively. The predictive model has better calibration power when the slope 
TABLE 1 | Basic characteristics of PORs in the training and validation cohorts.

\begin{tabular}{|c|c|c|c|}
\hline Characteristics & Training set $(n=179)$ & Validation set $(n=102)$ & $p$-Value \\
\hline Age (years) & $38(34-41)$ & $37(33-41)$ & 0.054 \\
\hline Stimulation protocols & & & 0.197 \\
\hline Pituitary downregulation (\%) & $35.75(64 / 179)$ & $44.12(45 / 102)$ & \\
\hline Non-pituitary downregulation (\%) & $64.25(115 / 179)$ & $56.88(57 / 102)$ & \\
\hline $\mathrm{BMI}\left(\mathrm{kg} / \mathrm{m}^{2}\right)$ & $22.46(20.19-24.46)$ & 21.87 (19.83-21.15) & 0.182 \\
\hline Type of infertility & & & 0.633 \\
\hline Primary infertility (\%) & $40.22(72 / 179)$ & $43.14(44 / 102)$ & \\
\hline Secondary infertility (\%) & $59.78(107 / 179)$ & $56.68(58 / 102)$ & \\
\hline Duration of infertility (years) & $3(1-5)$ & $3(2-5)$ & 0.958 \\
\hline Basal FSH (mlU/ml) & $8.59(7.08-10.77)$ & $8.48(7.05-9.75)$ & 0.085 \\
\hline Basal FSH/basal LH & $2.50 \pm 1.00$ & $2.31 \pm 0.85$ & 0.093 \\
\hline Basal E2 (pg/ml) & $41(29-57)$ & $44(33-57)$ & 0.686 \\
\hline $\mathrm{AMH}(\mathrm{ng} / \mathrm{ml})$ & $0.83(0.54-1.23)$ & $0.89(0.56-1.06)$ & 0.400 \\
\hline E2 on hCG day (pg/ml) & $1,563(912-2,450)$ & $1,851(1,228-2,605)$ & 0.058 \\
\hline LH on hCG day (mlU/ml) & $1.82(1.13-2.85)$ & $2.12(1.36-3.27)$ & 0.162 \\
\hline P on hCG day (ng/ml) & $0.84(0.60-1.53)$ & $0.92(0.69-1.36)$ & 0.070 \\
\hline AFC (n) & $6(4-7)$ & $6(5-7)$ & 0.664 \\
\hline Gonadotropin dose (IU) & $2,960.75 \pm 1,099.84$ & $3,150.37 \pm 942.36$ & 0.145 \\
\hline Gonadotropin days (days) & $9(8-11)$ & $10(9-12)$ & $0.040^{\star}$ \\
\hline Endometrial thickness (mm) & $10(9-12)$ & $10(9-12)$ & 0.7077 \\
\hline Type of endometrium & & & 0.667 \\
\hline Type A (\%) & $37.43(67 / 179)$ & $34.31(35 / 102)$ & \\
\hline Type B or C (\%) & $62.57(112 / 179)$ & 65.05 (67/102\%) & \\
\hline Oocytes retrieved (n) & $4(2-7)$ & $5(4-7)$ & 0.054 \\
\hline Mll oocytes (n) & $3(2-6)$ & $4(3-6)$ & 0.051 \\
\hline High-quality embryos (n) & $1(1-3)$ & $2(1-3)$ & 0.157 \\
\hline Clinical pregnancy (\%) & $34.08(61 / 179)$ & $43.14(44 / 102)$ & 0.131 \\
\hline
\end{tabular}

Pituitary downregulation means GnRH agonist long protocol. Non-pituitary downregulation includes GnRH antagonist protocol and the mild ovarian stimulation protocol. Continuous variables are shown as the median (interquartile range) or mean \pm standard deviation. Categorical variables are presented as percent.

BMI, body mass index; FSH, follicle-stimulating hormone; LH, luteinizing hormone; P, progesterone; E2, estradiol; AMH, anti-Müllerian hormone; hCG, human chorionic gonadotrophin; hCG E2, hCG LH, or hCG P means E2, LH, or P on the day of hCG administration; AFC, antral follicle count; endometrial thickness, the endometrial thickness on the day of hCG injection. *Training set vs. validation set: $p<0.05$.

TABLE 2 | Univariate analysis in the training group.

\begin{tabular}{lcc}
\hline Variables & OR $\mathbf{( 9 5 \% ~ C l )}$ & p-Value \\
\hline Age $>35$ (years) & $3.77(1.92-7.53)$ & $<0.001$ \\
Non-pituitary downregulation protocol & $1.28(0.65-2.48)$ & 0.469 \\
BMI $>24\left(\mathrm{~kg} / \mathrm{m}^{2}\right)$ & $2.21(1.12-4.52)$ & 0.025 \\
Secondary infertility & $1.26(0.65-2.43)$ & 0.485 \\
Duration of infertility $>3$ (years) & $1.37(0.71-2.70)$ & 0.353 \\
Basal FSH $>10(\mathrm{mlU} / \mathrm{ml})$ & $2.07(1.04-4.31)$ & 0.043 \\
Basal FSH/basal LH $>3$ & $0.658(0.34-1.31)$ & 0.225 \\
Basal E2 $>60(\mathrm{pg} / \mathrm{ml})$ & $2.03(0.99-4.38)$ & 0.061 \\
AMH $<0.7$ (ng/ml) & $1.76(0.87-3.74)$ & 0.125 \\
$\mathrm{P}$ on hCG day $>0.8(\mathrm{ng} / \mathrm{ml})$ & $0.71(0.37-1.36)$ & 0.305 \\
AFC $\leq 5$ (n) & $0.58(0.30-1.11)$ & 0.103 \\
Endometrial thickness $\leq 7$ (mm) & $1.11(0.43-3.24)$ & 0.845 \\
Type B or C of endometrium & $2.73(1.41-5.35)$ & 0.003 \\
Oocytes retrieved $\leq 3(\mathrm{n})$ & $1.61(0.82-3.26)$ & 0.173 \\
MII oocytes $\leq 3(\mathrm{n})$ & $1.78(0.93-3.46)$ & 0.082 \\
High-quality embryos $<2$ (n) & $2.26(1.15-4.45)$ & 0.018 \\
\hline
\end{tabular}

$\mathrm{OR}$, odds ratio; $\mathrm{Cl}$, confidence interval; BMI, body mass index; $F S H$, follicle-stimulating hormone; $A M H$, anti-Müllerian hormone; hCG, human chorionic gonadotrophin; AFC, antral follicle count; MII, metaphase II; LH, luteinizing hormone.

is closer to 1.000. The Brier score of calibration curves in the training set and validation set is 0.160 and 0.175 , respectively, which shows that the model is well-calibrated.

Internal validation (Table 4) shows performance indices of the model corrected for optimism after 200 bootstrapped
TABLE 3 | Multivariate logistic regression model in the training set.

\begin{tabular}{lccc}
\hline Variables & $\begin{array}{c}\text { Regression } \\
\text { coefficients }\end{array}$ & OR (95\% Cl) & p-Value \\
\hline Age $>35($ years $)$ & 0.953 & $2.59(1.24-5.47)$ & 0.012 \\
$\mathrm{BMl}>24\left(\mathrm{~kg} / \mathrm{m}^{2}\right)$ & 1.169 & $3.22(1.45-7.58)$ & 0.005 \\
Basal FSH $>10(\mathrm{mlU} / \mathrm{ml})$ & 1.053 & $2.87(1.28-6.75)$ & 0.012 \\
Basal E2 > 60 (pg/ml) & 0.902 & $2.47(1.08-5.93)$ & 0.036 \\
Type B or C of & 0.906 & $2.47(1.18-5.24)$ & 0.017 \\
endometrium & & & \\
High-quality embryos $<2(\mathrm{n})$ & 0.806 & $2.24(1.02-4.96)$ & 0.045 \\
\hline
\end{tabular}

$\mathrm{OR}$, odds ratio; $\mathrm{Cl}$, confidence interval; $\mathrm{BMI}$, body mass index; $\mathrm{FSH}$, follicle-stimulating hormone.

samples. Overall, the predictive model performs well, even after correction for optimism.

The decision-curve analysis shows that the prediction model is the higher line on the decision curve, which indicates that the prediction model leads to a higher net benefit and greater clinical utility (Supplementary 1).

\section{DISCUSSION}

This predictive model we developed aims to predict the individual probability of failed clinical pregnancy for women 


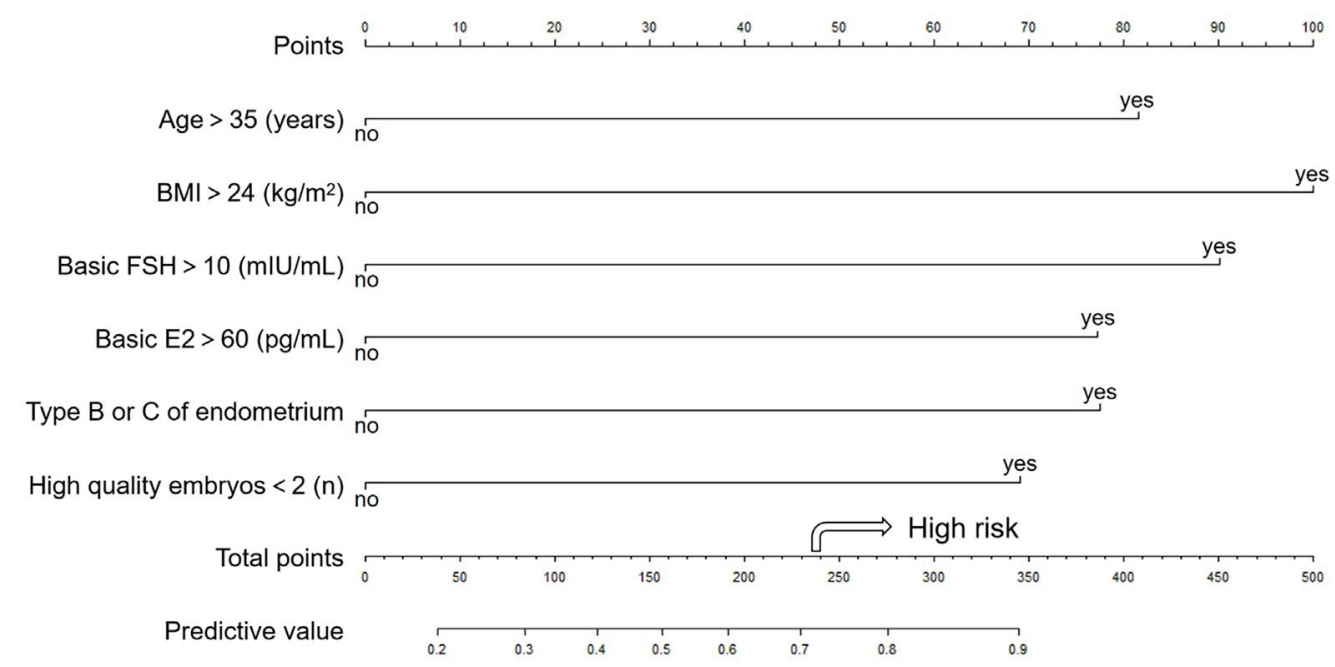

FIGURE 1 | The nomogram to predict the probability of clinical pregnancy failure in PORs. The nomogram can be applied by following procedures: (A) draw a line perpendicular from the corresponding axis of each risk factor until it reaches the top line labeled "Points"; (B) sum up the points for all risk factors and recorded as the total score; and (C) draw a line descending from the axis labeled "Total points" until it intercepts the lower line to determine the probability of failed conception. The arrow shows high risk when the total points are greater than 236.472. The optimal threshold point was calculated using receiver operating characteristic (ROC) curve.

A

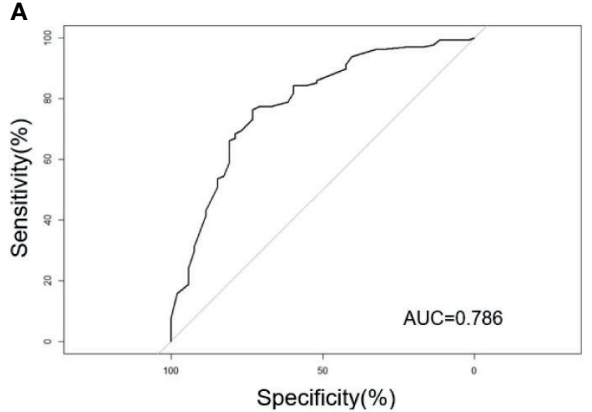

C

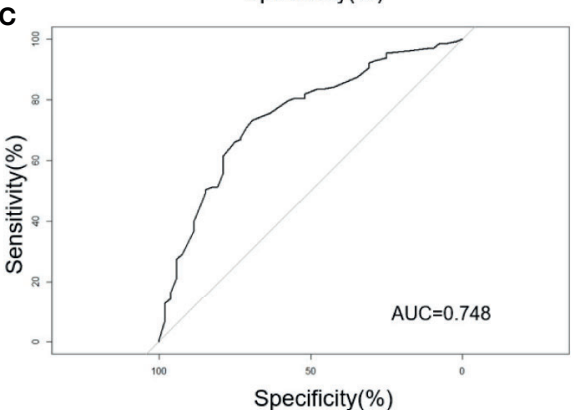

B

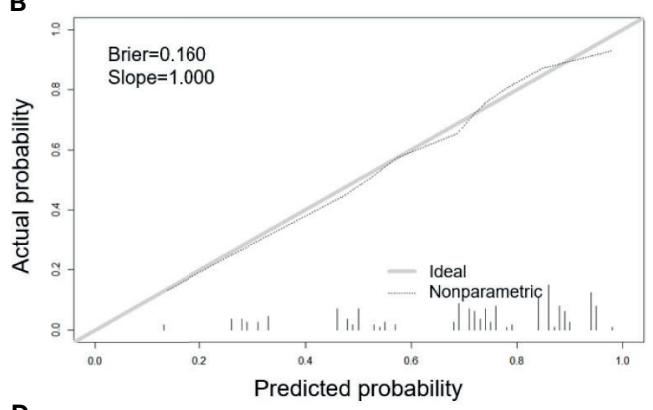

D

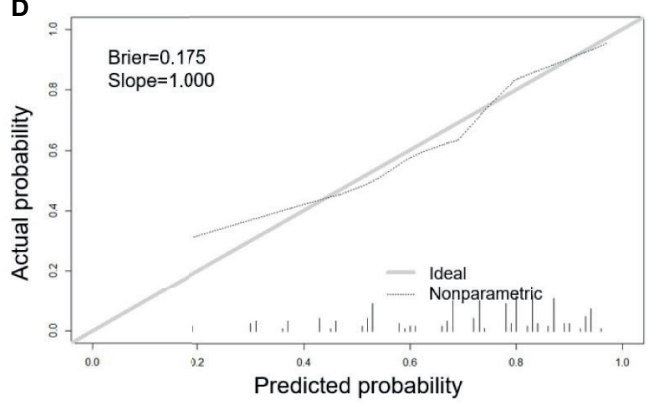

FIGURE 2 | Receiver operating characteristic (ROC) curves and calibration plots of the training and validation sets. (A) Area under the ROC curve (AUC) of the training set is 0.786 (95\% Cl: 0.710-0.861). (B) Calibration curve for training set (Brier $=0.160$, Slope = 1.000). (C) AUC of the validation set is 0.748 (95\% Cl: 0.668-0.827). (D) Calibration curve for validation set (Brier $=0.175$, Slope $=1.000$ ). Calibration curves were used to evaluate the calibration of the model. The horizontal axis is the predicted probability provided by this model, and the vertical axis is the observed incidence of pregnancy failure. The ideal line with $45^{\circ}$ slope represents a perfect prediction (the predicted probability equals the observed probability). The lower the Brier score for a set of predictions, the better the prediction calibration. When the slope was closer to 1.00, the prediction model had better calibration power.

with POR under IVF/ICSI-ET. The nomogram was developed in a training cohort including 179 PORs and tested on an external independent validation cohort including 102 patients with POR. Performance was evaluated using both calibration and discrimination. We have established three models at different time points for pregnancy failure prediction: model A (before ET), model B (before the start of IVF/ICSI cycle), and model C (on hCG day) (Supplementary 2, 3). After multiple 
comparisons, we chose the best-performing model A as our final prediction nomogram. Our nomogram is a user-friendly graphical representation of the model. The covariates of our model depend on the combination of readily available clinical and biological characteristics including patient age, BMI, basal E2, basal FSH, type of endometrium on hCG day, and the number of high-quality embryos, which are clinically significant and concordant with the published data.

There are a few models for predicting the success of clinical pregnancy of patients undergoing IVF/ICSI procedure in recent years $(15,16,26)$. In a pregnancy prediction model based on 1,675 IVF-double fresh embryo transfer cycles, there were no internal or external validations, and the predictive ability was relatively poor (26). A nomogram with good performance (AUC: $0.76)$ to predict the clinical pregnancy rate was only based on patients with endometriosis (16). Another model found independent predictors of the chance of clinical pregnancy after a completed IVF/ICSI cycle and did not focus on the special population of POR (15). Therefore, the advantages of our predictive model are as follows: first, it is a complete nomogram using a stepwise regression method with internal and external validations; second, the performance is quite good (AUC of 0.786); last but not least, it focuses on PORs, which is one of the high-risk groups associated with poor clinical pregnancy in IVF/ICSI cycles. We hypothesize that this nomogram can be used in the routine practice to facilitate physicians in predicting the pregnancy rate of PORs, selecting more appropriate individualized treatments, and helping patients with POR manage their expectations for conception.

In our model, maternal age $>35$ years is an independent risk factor of pregnancy failure. Patient age has been reported to be a vital prognostic factor in reproductive medicine and is frequently involved in evaluating the probability of pregnancy. Increased patient age is associated with decreased clinical pregnancy rate $(16,27,28)$. In an Australian cohort of 36,412 patients initiated with first autologous fresh IVF cycles, for women $\geq 30$ years, every 1 -year increase in age was associated with an $11 \%$ reduction in the chance of achieving pregnancy. If women aged 35 years or older would have had their first autologous fresh treatment 1 year earlier, $15 \%$ extra deliveries would be expected (29). The trend that older poor responders have a lower pregnancy rate compared with younger poor responders has been revealed in several researches $(10,12,30,31)$. For poor responders over 35 years, the rates of implantation and clinical pregnancy were lower than those of under 35. Meanwhile, younger poor responders $(<35)$ still have a reasonable number of transferable embryos $(2.02 \pm 0.57)$ and an acceptable

TABLE 4 | Performance of internal validation.

\begin{tabular}{lccc}
\hline Index & Original & Optimism & $\begin{array}{c}\text { Optimism-corrected } \\
\text { value }\end{array}$ \\
\hline Dxy & 0.571 & 0.051 & 0.520 \\
$\mathrm{R}^{2}$ & 0.281 & 0.054 & 0.228 \\
Brier & 0.160 & -0.013 & 0.173 \\
\hline
\end{tabular}

The original dataset was corrected for optimism with 200 bootstrap samples. pregnancy rate $(37.50 \%)$ (32). It is well understood that increased age leads to a reduction in the quantity and quality of oocytes, which is accompanied by a decline in female fertility $(33,34)$. Therefore, PORs should be treated positively, and IVF/ ICSI treatment should be considered earlier.

Overweight and obesity raise major challenges for women of reproductive age. Up to $60 \%$ of worldwide women are overweight, and up to $30 \%$ of these women are obese $(35,36)$. In a large retrospective study of 500,000 autologous IVF cycles, obese women had a $6 \%$ reduction in intrauterine pregnancy rates and a $13 \%$ reduction in live birth rates as compared with normalweight women (37). Obese poor responders might have a lower pregnancy rate than non-obese poor responders. One study described a significant decrease in pregnancy rate for the poor responders with BMI $>30 \mathrm{~kg} / \mathrm{m}^{2}$ versus $\mathrm{BMI} \leq 30 \mathrm{~kg} / \mathrm{m}^{2}(4.5 \%$ versus $23 \%$, respectively). However, non-obese PORs achieved pregnancy rates comparable with those of normal responders (38). Correlation analysis in our cohort revealed that both overweight status and obese status of PORs were related to receiving pregnancy failure and could be a predictor for adverse pregnancy outcomes. This is because impaired ovarian follicular genesis (39), oocyte quality (40,41), embryonic development $(40,42)$, and endometrial receptivity $(41,43)$ might be involved in poorer reproductive outcomes in obese women. Fortunately, high body weight is a reversible basic parameter. Weight loss of $10 \mathrm{~kg}$ over 6 months was shown to improve ovulation function and rates of conception in obese anovulatory women (44). Therefore, due to the high risk of low pregnancy rates in PORs, weight management should be encouraged for them in preconception counseling to potentially improve ART outcomes as well as reduce Gn dosage and anesthetic dosage during oocyte retrieval.

Our data indicated that increasing basal FSH levels were associated with lower pregnancy rates in women with POR. With a similar cutoff point, two studies found that the chance of pregnancy was significantly higher in women with basal FSH $<10$ IU/L than in women with FSH $\geq 10$ IU/L under IVF treatment $(26,45)$. In an analysis of 163 poor responders, the pregnancy rate for patients with an elevated basal FSH (>12.0 IU/L) was significantly decreased versus those with normal FSH $(4.0 \%$ versus $14.8 \%$, respectively) (30). As is well known, the FSH level shows a rising trend with increasing age. Some researchers hold an interesting point that younger ages seem to protect a woman against the negative effect of a raised FSH concentration $(45,46)$. This further suggests that PORs require ART as soon as possible to have more chance of conceiving, since ovarian responsiveness and clinical outcome deteriorate with increasing age and serum FSH. Meanwhile, basal serum FSH >10 IU/L indicates the DOR, which is closely related with the number and quality of retrieved oocytes. In this regard, the importance of basal FSH concentration would lie in developing a tailored stimulation protocol that can maximize the ovarian response by achieving a successful pregnancy outcome, especially in PORs. However, some other indicators of DOR (such as AMH and AFC) were not specific to screen for failure to conceive for PORs in our data. There is emerging evidence to support our findings 
that the low AMH cutoff points $(0.2-0.7 \mathrm{ng} / \mathrm{ml})$ or low AFC $(\leq 5)$ have moderate-to-high specificity as a screening test for POR but not specific for predicting pregnancy failure (47-50). Also, AMH and AFC are relatively low in a majority group of PORs, which may reduce the prediction performance.

An early rise in serum basal E2 concentration is a classic feature of reproductive aging. Baseline E2 level $>60 \mathrm{pg} / \mathrm{ml}$ is used as a risky predictor for pregnancy failure in our model. When the basal FSH concentration is normal but the serum estradiol level is above $60-80 \mathrm{pg} / \mathrm{ml}$ in the early follicular phase, there is some evidence of association with poor response, increased cancellation rates, and decreased pregnancy rates (51-53). We speculate that there are two reasonable explanations. On the one hand, DOR is responsible for poor pregnancy outcome. The diminished inhibin production from the pituitary increases basal FSH level and secondarily increases E2 production from granulosa cells in the ovary in the early follicular phase of women with decreased ovarian reserve and function. This in turn will suppress FSH production and release. A temporary balance between pituitary and ovarian response will result in normal FSH levels and increased circulating E2. On the other hand, the limited number of oocytes retrieved causes low pregnancy rates. High basal E2 hinders the development of the dominant follicle, which influences the ovarian response and decreases the total number of retrieved oocytes after stimulation.

In IVF/ICSI fresh ET cycles, high-quality embryos will be preferentially transferred into the uterine cavity because good quality is associated with high success rate of clinical pregnancy. In our model, the number of high-quality embryos below 2 boosts pregnancy failure risk. It is comprehensible that in the two transferred embryos per fresh cycle, both of them belong to grade I or II and will be more likely to implant into the endometrium. Therefore, women with POR take greater risks of transfer cancellation to accumulate good embryos. The endometrial pattern also predicts the pregnancy outcome in our model. Pattern A with complete triple line at ultrasound examination reflects endometrial proliferation. This presence on the day of hCG injection is associated with a higher pregnancy rate than the absence of this pattern (54), which is consistent with our conclusion. However, whether endometrial thickness affects pregnancy is controversial. One study summarized that similar clinical pregnancy rates were found between women with triple-line pattern and women without triple-line pattern assessed on the day of hCG undergoing IVF with fresh ET (55). The absence of a triple-line pattern may be a sign of premature secretory changes of the endometrium and the passed time-window of maximal endometrial receptivity (56). This status is not conducive to embryo implantation. However, whether endometrial thickness affects pregnancy is controversial. One study summarized that similar clinical pregnancy rates were found between women with triple-line pattern and women without triple-line pattern assessed on the day of hCG undergoing IVF with fresh ET (55). Further data relevant to the clinical value of the endometrial patterns are needed.

The main limitation of our model is its retrospective nature, which cannot exclude all potential biases. Besides, the data collection is based on our single center, and no independent external validation cohorts from other hospitals were included in our study. Furthermore, due to the high cancellation rates of PORs, further studies concerning the predictive factors for cumulative pregnancy after fresh and frozen-thawed ET cycles are needed. Prospective, large-scale, and multicenter clinical trials should be carried out in the future.

\section{CONCLUSION}

In conclusion, our analysis resulted in a well-calibrated model that can predict the risk of clinical pregnancy failure in PORs under IVF/ICSI-ET cycles to help physicians choose more appropriate individualized treatments and to help patients with POR to manage their expectations for ART outcomes.

\section{DATA AVAILABILITY STATEMENT}

The datasets presented in this article are not readily available because This database relates to the confidentiality of our clinical center and patient privacy, so it is not convenient to disclose. Requests to access the datasets should be directed to FL, lfyhelen24@163.com.

\section{ETHICS STATEMENT}

The studies involving human participants were reviewed and approved by Biomedical Research Ethics Committee of Peking University First Hospital. The patients/participants provided their written informed consent to participate in this study.

\section{AUTHOR CONTRIBUTIONS}

(I) Conception and design: FL, CZ, QX. (II) Administrative support: QX. (III) Provision of study materials or patients: XL, QX. (IV) Collection and assembly of data: FL, XL. (V) Data analysis and interpretation: FL, RL. (VI) Manuscript writing: FL; (VII) Final approval of manuscript: CZ, QX. All authors contributed to the article and approved the submitted version.

\section{FUNDING}

National Natural Science Foundation of China Major Research Program Cultivation Project (91949113).

\section{SUPPLEMENTARY MATERIAL}

The Supplementary Material for this article can be found online at: https://www.frontiersin.org/articles/10.3389/fendo.2021. 717288/full\#supplementary-material 


\section{REFERENCES}

1. La Marca A, Sunkara SK. Individualization of Controlled Ovarian Stimulation in IVF Using Ovarian Reserve Markers: From Theory to Practice. Hum Reprod Update (2014) 20(1):124-40. doi: 10.1093/humupd/dmt037

2. Ozkan ZS. Ovarian Stimulation Modalities in Poor Responders. Turk J Med Sci (2019) 49(4):959-62. doi: 10.3906/sag-1905-179

3. Bosdou JK, Venetis CA, Kolibianakis EM, Toulis KA, Goulis DG, Zepiridis L, et al. The Use of Androgens or Androgen-Modulating Agents in Poor Responders Undergoing In Vitro Fertilization: A Systematic Review and Meta-Analysis. Hum Reprod Update (2012) 18(2):127-45. doi: 10.1093/ humupd/dmr051

4. Kyrou D, Kolibianakis EM, Venetis CA, Papanikolaou EG, Bontis J, Tarlatzis BC. How to Improve the Probability of Pregnancy in Poor Responders Undergoing In Vitro Fertilization: A Systematic Review and Meta-Analysis. Fertil Steril (2009) 91(3):749-66. doi: 10.1016/j.fertnstert.2007.12.077

5. Keay SD. Poor Ovarian Response to Gonadotrophin Stimulation the Role of Adjuvant Treatments. Hum Fertil (Camb) (2002) 5(1 Suppl):S46-52. doi: 10.1080/1464727022000199921

6. Yilmaz N, Uygur D, Inal H, Gorkem U, Cicek N, Mollamahmutoglu L. Dehydroepiandrosterone Supplementation Improves Predictive Markers for Diminished Ovarian Reserve: Serum AMH, Inhibin B and Antral Follicle Count. Eur J Obstet Gynecol Reprod Biol (2013) 169(2):257-60. doi: 10.1016/ j.ejogrb.2013.04.003

7. Badawy A, Wageah A, El Gharib M, Osman EE. Prediction and Diagnosis of Poor Ovarian Response: The Dilemma. J Reprod Infertil (2011) 12(4):241-8.

8. Ubaldi F, Vaiarelli A, D’Anna R, Rienzi L. Management of Poor Responders in IVF: Is There Anything New? BioMed Res Int (2014) 2014:1-10. doi: $10.1155 / 2014 / 352098$

9. Keay SD, Liversedge NH, Mathur RS, Jenkins JM. Assisted Conception Following Poor Ovarian Response to Gonadotrophin Stimulation. Br J Obstet Gynaecol (1997) 104(5):521-7. doi: 10.1111/j.1471-0528.1997.tb11525.x

10. Oudendijk JF, Yarde F, Eijkemans MJ, Broekmans FJ, Broer SL. The Poor Responder in IVF: Is the Prognosis Always Poor?: A Systematic Review. Hum Reprod Update (2012) 18(1):1-11. doi: 10.1093/humupd/dmr037

11. Surrey ES, Schoolcraft WB. Evaluating Strategies for Improving Ovarian Response of the Poor Responder Undergoing Assisted Reproductive Techniques. Fertil Steril (2000) 73(4):667-76. doi: 10.1016/s0015-0282(99) 00630-5

12. Zhen XM, Qiao J, Li R, Wang LN, Liu P. The Clinical Analysis of Poor Ovarian Response in in-Vitro-Fertilization Embryo-Transfer Among Chinese Couples. J Assist Reprod Genet (2008) 25(1):17-22. doi: 10.1007/s10815-007-9187-9

13. Zhang M, Niu W, Wang Y, Xu J, Bao X, Wang L, et al. Dehydroepiandrosterone Treatment in Women With Poor Ovarian Response Undergoing IVF or ICSI: A Systematic Review and Meta-Analysis. J Assist Reprod Genet (2016) 33(8):981-91. doi: 10.1007/s10815-016-0713-5

14. Roberts SA, Hirst WM, Brison DR, Vail A. Toward SEtc. Embryo and Uterine Influences on IVF Outcomes: An Analysis of a UK Multi-Centre Cohort. Hum Reprod (2010) 25(11):2792-802. doi: 10.1093/humrep/deq213

15. Cai QF, Wan F, Huang R, Zhang HW. Factors Predicting the Cumulative Outcome of IVF/ICSI Treatment: A Multivariable Analysis of 2450 Patients. Hum Reprod (2011) 26(9):2532-40. doi: 10.1093/humrep/der228

16. Ballester M, Oppenheimer A, d'Argent EM, Touboul C, Antoine JM, Coutant C, et al. Nomogram to Predict Pregnancy Rate After ICSI-IVF Cycle in Patients With Endometriosis. Hum Reprod (2012) 27(2):451-6. doi: 10.1093/ humrep/der392

17. Ferraretti AP, La Marca A, Fauser BC, Tarlatzis B, Nargund G, Gianaroli L, et al. ESHRE Consensus on the Definition of 'Poor Response' to Ovarian Stimulation for In Vitro Fertilization: The Bologna Criteria. Hum Reprod (2011) 26(7):1616-24. doi: 10.1093/humrep/der092

18. Chen CM. Overview of Obesity in Mainland China. Obes Rev (2008) 9 Suppl 1:14-21. doi: 10.1111/j.1467-789X.2007.00433.x

19. von Wolff M, Schwartz AK, Bitterlich N, Stute P, Fah M. Only Women's Age and the Duration of Infertility Are the Prognostic Factors for the Success Rate of Natural Cycle IVF. Arch Gynecol Obstet (2019) 299(3):883-9. doi: 10.1007/ s00404-018-5034-8

20. Committee opinion no. 618. Ovarian Reserve Testing. Obstet Gynecol (2015) 125(1):268-73. doi: 10.1097/01.AOG.0000459864.68372.ec
21. Atasever M, Soyman Z, Demirel E, Gencdal S, Kelekci S. Diminished Ovarian Reserve: Is it a Neglected Cause in the Assessment of Recurrent Miscarriage? A Cohort Study. Fertil Steril (2016) 105(5):1236-40. doi: 10.1016/ j.fertnstert.2016.01.001

22. Seckin B, Turkcapar F, Ozaksit G. Elevated Day 3 FSH/LH Ratio: A Marker to Predict IVF Outcome in Young and Older Women. J Assist Reprod Genet (2012) 29(3):231-6. doi: 10.1007/s10815-011-9695-5

23. Yuan X, Saravelos SH, Wang Q, Xu Y, Li TC, Zhou C. Endometrial Thickness as a Predictor of Pregnancy Outcomes in 10787 Fresh IVF-ICSI Cycles. Reprod BioMed Online (2016) 33(2):197-205. doi: 10.1016/j.rbmo.2016.05.002

24. Venetis CA, Kolibianakis EM, Bosdou JK, Tarlatzis BC. Progesterone Elevation and Probability of Pregnancy After IVF: A Systematic Review and Meta-Analysis of Over 60000 Cycles. Hum Reprod Update (2013) 19(5):43357. doi: 10.1093/humupd/dmt014

25. Youden WJ. Index for Rating Diagnostic Tests. Cancer (1950) 3(1):32-5. doi: 10.1002/1097-0142(1950)3:1<32::aid-cncr2820030106>3.0.co;2-3

26. Ottosen LD, Kesmodel U, Hindkjaer J, Ingerslev HJ. Pregnancy Prediction Models and eSET Criteria for IVF Patients-Do We Need More Information? J Assist Reprod Genet (2007) 24(1):29-36. doi: 10.1007/s10815-006-9082-9

27. Gonzalez-Foruria I, Penarrubia J, Borras A, Manau D, Casals G, Peralta S, et al. Age, Independent From Ovarian Reserve Status, Is the Main Prognostic Factor in Natural Cycle In Vitro Fertilization. Fertil Steril (2016) 106(2):342347 e342. doi: 10.1016/j.fertnstert.2016.04.007

28. Gao L, Li M, Wang Y, Zeng Z, Xie Y, Liu G, et al. Overweight and High Serum Total Cholesterol Were Risk Factors for the Outcome of IVF/ICSI Cycles in PCOS Patients and a PCOS-Specific Predictive Model of Live Birth Rate was Established. J Endocrinol Invest (2020) 43(9):1221-8. doi: 10.1007/s40618-020-01209-5

29. Wang YA, Healy D, Black D, Sullivan EA. Age-Specific Success Rate for Women Undertaking Their First Assisted Reproduction Technology Treatment Using Their Own Oocytes in Australia, 2002-2005. Hum Reprod (2008) 23(7):1633-8. doi: 10.1093/humrep/den135

30. Galey-Fontaine J, Cedrin-Durnerin I, Chaibi R, Massin N, Hugues JN. Age and Ovarian Reserve Are Distinct Predictive Factors of Cycle Outcome in Low Responders. Reprod BioMed Online (2005) 10(1):94-9. doi: 10.1016/s14726483(10)60808-5

31. Kilic S, Yilmaz N, Zulfikaroglu E, Sarikaya E, Kose K, Topcu O, et al. Obesity Alters Retrieved Oocyte Count and Clinical Pregnancy Rates in High and Poor Responder Women After In Vitro Fertilization. Arch Gynecol Obstet (2010) 282(1):89-96. doi: 10.1007/s00404-010-1359-7

32. Gong Y, Li-Ling J, Xiong D, Wei J, Zhong T, Tan H. Age-Related Decline in the Expression of GDF9 and BMP15 Genes in Follicle Fluid and Granulosa Cells Derived From Poor Ovarian Responders. J Ovarian Res (2021) 14(1):1. doi: 10.1186/s13048-020-00757-x

33. Tufan E, Elter K, Durmusoglu F. Assessment of Reproductive Ageing Patterns by Hormonal and Ultrasonographic Ovarian Reserve Tests. Hum Reprod (2004) 19(11):2484-9. doi: 10.1093/humrep/deh448

34. Baird DT, Collins J, Egozcue J, Evers LH, Gianaroli L, Leridon H, et al. Fertility and Ageing. Hum Reprod Update (2005) 11(3):261-76. doi: 10.1093/humupd/dmi006

35. Haslam DW, James WPT. Obesity. Lancet (2005) 366(9492):1197-209. doi: 10.1016/s0140-6736(05)67483-1

36. Obesity: Preventing and Managing the Global Epidemic. Report of a WHO Consultation. World Health Organ Tech Rep Ser (2000) 894:i-xii, 1-253.

37. Kawwass JF, Kulkarni AD, Hipp HS, Crawford S, Kissin DM, Jamieson DJ. Extremities of Body Mass Index and Their Association With Pregnancy Outcomes in Women Undergoing In Vitro Fertilization in the United States. Fertil Steril (2016) 106(7):1742-50. doi: 10.1016/j.fertnstert.2016.08.028

38. Orvieto R, Meltcer S, Nahum R, Rabinson J, Anteby EY, Ashkenazi J. The Influence of Body Mass Index on In Vitro Fertilization Outcome. Int J Gynaecol Obstet (2009) 104(1):53-5. doi: 10.1016/j.ijgo.2008.08.012

39. Jungheim ES, Moley KH. Current Knowledge of Obesity's Effects in the Preand Periconceptional Periods and Avenues for Future Research. Am J Obstet Gynecol (2010) 203(6):525-30. doi: 10.1016/j.ajog.2010.06.043

40. Catteau A, Caillon H, Barriere P, Denis MG, Masson D, Freour T. Leptin and its Potential Interest in Assisted Reproduction Cycles. Hum Reprod Update (2016) 22(3):320-41. doi: 10.1093/humupd/dmv057

41. Broughton DE, Moley KH. Obesity and Female Infertility: Potential Mediators of Obesity's Impact. Fertil Steril (2017) 107(4):840-7. doi: 10.1016/ j.fertnstert.2017.01.017 
42. Comstock IA, Kim S, Behr B, Lathi RB. Increased Body Mass Index Negatively Impacts Blastocyst Formation Rate in Normal Responders Undergoing In Vitro Fertilization. J Assist Reprod Genet (2015) 32(9):1299-304. doi: 10.1007/ s10815-015-0515-1

43. Rhee JS, Saben JL, Mayer AL, Schulte MB, Asghar Z, Stephens C, et al. DietInduced Obesity Impairs Endometrial Stromal Cell Decidualization: A Potential Role for Impaired Autophagy. Hum Reprod (2016) 31(6):1315-26. doi: 10.1093/humrep/dew048

44. Clark AM, Thornley B, Tomlinson L, Galletley C, Norman RJ. Weight Loss in Obese Infertile Women Results in Improvement in Reproductive Outcome for All Forms of Fertility Treatment. Hum Reprod (1998) 13(6):1502-5. doi: 10.1093/humrep/13.6.1502

45. Sabatini L, Zosmer A, Hennessy EM, Tozer A, Al-Shawaf T. Relevance of Basal Serum FSH to IVF Outcome Varies With Patient Age. Reprod BioMed Online (2008) 17(1):10-9. doi: 10.1016/s1472-6483(10)60287-8

46. Roberts JE, Spandorfer S, Fasouliotis SJ, Kashyap S, Rosenwaks Z. Taking a Basal Follicle-Stimulating Hormone History Is Essential Before Initiating In Vitro Fertilization. Fertil Steril (2005) 83(1):37-41. doi: 10.1016/j.fertnstert.2004.06.062

47. Hendriks DJ, Mol BW, Bancsi LF, Te Velde ER, Broekmans FJ. Antral Follicle Count in the Prediction of Poor Ovarian Response and Pregnancy After In Vitro Fertilization: A Meta-Analysis and Comparison With Basal FollicleStimulating Hormone Level. Fertil Steril (2005) 83(2):291-301. doi: 10.1016/ j.fertnstert.2004.10.011

48. Gnoth C, Schuring AN, Friol K, Tigges J, Mallmann P, Godehardt E. Relevance of Anti-Mullerian Hormone Measurement in a Routine IVF Program. Hum Reprod (2008) 23(6):1359-65. doi: 10.1093/humrep/den108

49. Penarrubia J, Fabregues F, Manau D, Creus M, Casals G, Casamitjana R, et al. Basal and Stimulation Day 5 Anti-Mullerian Hormone Serum Concentrations as Predictors of Ovarian Response and Pregnancy in Assisted Reproductive Technology Cycles Stimulated With Gonadotropin-Releasing Hormone Agonist-Gonadotropin Treatment. Hum Reprod (2005) 20(4):915-22. doi: 10.1093/humrep/deh718

50. Ficicioglu C, Kutlu T, Baglam E, Bakacak Z. Early Follicular Antimullerian Hormone as an Indicator of Ovarian Reserve. Fertil Steril (2006) 85(3):592-6. doi: 10.1016/j.fertnstert.2005.09.019

51. Evers JL, Slaats P, Land JA, Dumoulin JC, Dunselman GA. Elevated Levels of Basal Estradiol-17beta Predict Poor Response in Patients With Normal Basal
Levels of Follicle-Stimulating Hormone Undergoing In Vitro Fertilization. Fertil Steril (1998) 69(6):1010-4. doi: 10.1016/s0015-0282(98)00080-6

52. Licciardi FL, Liu H-C, Rosenwaks Z. Day 3 Estradiol Serum Concentrations as Prognosticators of Ovarian Stimulation Response and Pregnancy Outcome in Patients Undergoing In Vitro Fertilization ${ }^{* *}$ Presented in Part at the Annual Meeting of the Society of Gynecologic Investigation, San Antonio, Texas, March 11 to 14, 1991. Fertil Steril (1995) 64(5):991-4. doi: 10.1016/s00150282(16)57916-3

53. Smotrich DB, Widra EA, Gindoff PR, Levy MJ, Hall JL, Stillman RJ. Prognostic Value of Day 3 Estradiol on In Vitro Fertilization Outcome. Fertil Steril (1995) 64(6):1136-40. doi: 10.1016/S0015-0282(16)57974-6

54. Zhao J, Zhang Q, Wang Y, Li Y. Endometrial Pattern, Thickness and Growth in Predicting Pregnancy Outcome Following 3319 IVF Cycle. Reprod BioMed Online (2014) 29(3):291-8. doi: 10.1016/j.rbmo.2014.05.011

55. Craciunas L, Gallos I, Chu J, Bourne T, Quenby S, Brosens JJ, et al. Conventional and Modern Markers of Endometrial Receptivity: A Systematic Review and Meta-Analysis. Hum Reprod Update (2019) 25 (2):202-23. doi: 10.1093/humupd/dmy044

56. Bourgain C, Devroey P. The Endometrium in Stimulated Cycles for IVF. Hum Reprod Update (2003) 9(6):515-22. doi: 10.1093/humupd/dmg045

Conflict of Interest: The authors declare that the research was conducted in the absence of any commercial or financial relationships that could be construed as a potential conflict of interest.

Publisher's Note: All claims expressed in this article are solely those of the authors and do not necessarily represent those of their affiliated organizations, or those of the publisher, the editors and the reviewers. Any product that may be evaluated in this article, or claim that may be made by its manufacturer, is not guaranteed or endorsed by the publisher.

Copyright (c) $2021 \mathrm{Li}, \mathrm{Lu}$, Zeng, Li and Xue. This is an open-access article distributed under the terms of the Creative Commons Attribution License (CC BY). The use, distribution or reproduction in other forums is permitted, provided the original author(s) and the copyright owner(s) are credited and that the original publication in this journal is cited, in accordance with accepted academic practice. No use, distribution or reproduction is permitted which does not comply with these terms. 